ISSN 1997-342X (Online), ISSN 1991-8631 (Print)

Original Paper http://ajol.info/index.php/ijbcs http://indexmedicus.afro.who.int

\title{
Evaluation de la qualité des eaux des ruisseaux du cours moyen de la rivière Alibori par l'étude des macroinvertébrés benthiques dans le bassin cotonnier du Bénin (Afrique de l'Ouest)
}

\author{
Thierry M. AGBLONON HOUELOME ${ }^{1 *}$, Delphine ADANDEDJAN ${ }^{1}$, \\ Antoine CHIKOU ${ }^{1}$, Ibrahim IMOROU TOKO ${ }^{2}$, Clément BONOU $^{3}$, \\ Issaka YOUSSAO $^{4}$ et Philippe LALEYE ${ }^{1}$
}

\begin{abstract}
1 Laboratoire d'Hydrobiologie et d'Aquaculture, Faculté des Sciences Agronomiques, Université d'AbomeyCalavi (LHA/FSA/UAC), 01 BP : 526 Cotonou, Bénin.

2 Unité de Recherche en Aquaculture et Ecotoxicologie Aquatique (URAEAq), Faculté d'Agronomie, Université de Parakou, 03 BP : 61 Parakou-URAEAq, Bénin.

3 Département de l'Aménagement et Protection de l'Environnement, Ecole Polytechnique d'Abomey-Calvi, Université d'Abomey-Calvi (APE/EPAC/UAC), 01 BP 2009 Cotonou; Bénin.

4 Département de Production et Santé Animales, Ecole Polytechnique d'Abomey-Calvi, Université d'AbomeyCalvi (PSA/EPAC/UAC), 01 BP 2009 Cotonou; Bénin.

*Auteur correspondant ; E-mail: tagblonon@gmail.com,Tel :00229 67118979.
\end{abstract}

\section{RESUME}

L'impact des pressions anthropiques sur les écosystèmes aquatiques du bassin cotonnier du Bénin a été évalué d'octobre à décembre 2012. L'objectif est de déterminer la qualité écologique des ruisseaux du bassin de la rivière Alibori par l'étude des peuplements de macroinvertébrés. Au moyen d'un filet troubleau, les macroinvertébrés ont été échantillonnés dans 12 stations réparties dans les ruisseaux du bassin moyen de la rivière. L'inventaire de la macrofaune benthique récoltée a permis de disposer de 25 espèces d'invertébrés aquatiques appartenant à 16 familles et 7 ordres. Ils sont constitués de trois classes qui sont les Insectes $(5$ ordres, 12 familles et 20 espèces), les Gastéropodes ( 1 ordre, 3 familles et 4 espèces) et les Lamellibranches (Bivalves) ( 1 ordre, 1 famille et 1 espèce). L'analyse de la structure de la communauté des taxons grâce à SOM sur la base de la matrice de présence-absence a révélé une répartition spatiale indiquant que les différentes stations ont eu en commun une grande partie de la faune benthique mais se sont différenciées par un nombre limité de taxons qui ont caractérisé chaque station. Six paramètres environnementaux ont discriminé cette répartition observée. L'abondance de certains groupes benthiques comme les Diptères et les Coléoptères dans les ruisseaux montre que le bassin cotonnier du Bénin est soumis aux perturbations anthropiques.

(C) 2016 International Formulae Group. All rights reserved.

Mots clés : Macroinvertébrés benthiques, rivière Alibori, ruisseaux, perturbations. 


\title{
Assessment of the quality of streams water in the middle course of the Alibori river through the study of benthic macroinvertebrates in the cotton basin of Benin (West Africa)
}

\begin{abstract}
The impact of the anthropic activities on the water ecosystems of the Benin cotton basin was assessed from October to December. The objective of this work is to determine the streams quality of Alibori basin by studying of benthic macroinvertebrates. A sample of macroinvertebrates was collected at 12 stations in the streams of the basin of the Alibori River. The inventory of the collected benthic macrofauna displayed a total of 25 species of water invertebrates belonging 16 families and 7 orders. These orders consisted of three classes: the Insects (5 orders, 12 families and 20 species), the Gastropods (1 order, 3 families and 4 species) and Bivalve ( 1 order, 1 family and 1 species). The analysis of the presence-absence of taxa obtained through SOM revealed a space distribution which indicates that the various sampling stations had in common a great part of benthic fauna and that their differences lie in a limited number of taxa which were specific to each sampling site. Six environmental parameters have discriminated this observed distribution. The abundance of some benthic groups such as Diptera and Coleoptera in the streams during the period of study shows that this basin is subject to anthropogenic disturbances.
\end{abstract}

(C) 2016 International Formulae Group. All rights reserved.

Keywords: Benthic macroinvertebrates, Alibori river, streams, disturbances.

\section{INTRODUCTION}

L'intensification des activités agricoles constitue la cause majeure de dégradation des écosystèmes aquatiques en milieu rural (Leight et al., 2010 ; Sass et al., 2010). Les activités agricoles sur les bassins versants présentent de sérieuses répercussions écologiques pour les plans et cours d'eau (Hepp et al., 2010). En effet, l'usage des pesticides et engrais chimiques expriment en sourdine des effets néfastes sur les communautés biologiques aquatiques. Après l'utilisation phytosanitaire, ces intrants agricoles se dispersent dans les différents compartiments de l'environnement. Seuls $0,1 \%$ des pesticides pulvérisés dans les champs atteignent leur cible, le reste se répartit dans les écosystèmes pour contaminer l'air, la terre et l'eau (Agbohessi et al., 2013). Le compartiment aquatique, constituant le réceptacle ultime des résidus, représente le milieu le plus affecté. Ces produits dangereux détériorent les peuplements, engendrent des intoxications, induisent des perturbations biocénotiques et conduisent même parfois à l'extinction de certaines espèces (YounesBaraillé et al., 2005 ; Agbohessi et al., 2013).

De nos jours, il a été admis que les systèmes d'évaluation de la qualité des eaux de surface doivent tenir compte des critères biologiques (Fishar et Williams, 2008) c'està-dire de bioindicateurs. Ainsi, ce sont les organismes macrobenthiques qui sont les groupes d'organismes les plus utilisés comme bioindicateurs (Clarke et al., 2002; Gnohossou, 2006; Edia, 2008; Adandédjan, 2012). Leur étude permet d'évaluer les répercussions réelles de la pollution et de l'altération des habitats aquatiques et riverains (Touzin, 2008; Adandédjan, 2012). D'où l'importance d'évaluer la qualité des eaux des ruisseaux de la rivière Alibori qui constituent les principaux véhicules des pesticides et les engrais utilisés en culture cotonnière vers le lit de la rivière, par l'étude des macroinvertébrés. L'étude vise à acquérir de meilleures connaissances sur les peuplements macrobenthiques du bassin cotonnier de la 
rivière Alibori afin d'apprécier leur niveau de perturbation.

\section{MATERIEL ET METHODES \\ Milieu d'étude}

La rivière Alibori est un écosystème situé en zone soudanienne entre $10^{\circ} 30^{\prime}$ et $12^{\circ}$ de latitude Nord et $1^{\circ} 32^{\prime}$ et $3^{\circ} 50^{\prime}$ de longitude Est. Elle naît à $410 \mathrm{~m}$ d'altitude environ, dans le massif granitique de Kita sur le flanc de la chaîne de l'Atacora dans la commune de Péhonco. D'un bassin versant couvrant une superficie de $13740 \mathrm{~km}^{2}$, la rivière Alibori est longue de $427 \mathrm{~km}$. Dans son cours moyen, le lit de la rivière est coupé par de nombreux rapides. La pente du lit de l'Alibori est dans l'ensemble faible, de 0,5 à $0,2 \mathrm{~m} / \mathrm{km}$. Le bassin de l'Alibori est bordé, à l'Ouest, par les reliefs correspondant aux roches les plus basiques du groupe de Kandi; à l'Est, par les collines quartzitiques du groupe de Gbadagba dans la région de Bembèrèkè, puis par les reliefs formés sur les roches du groupe de Kandi. L'Alibori reçoit sur sa rive droite le Souédarou, le Sarédarou et le Darou-Woka; sur sa rive gauche, le Morokou, la Kparé, le Kénou, le Konékoga et le Kpako avant de se jeter dans le Niger en amont de Malanville (Le Barbe et al., 1993 et Bio Bigou, 1987). Elle s'étend dans le département d'Alibori avec beaucoup d'affluents éparpillés dans les communes de Kandi, Gogounou, Banikora, Ségbana, Karimama et Malanville.

Le climat dans cette région correspond à une zone soudanienne sèche de type semiaride avec une saison pluvieuse et une saison sèche. La région est arrosée avec une pluviométrie de hauteur 700 à $1200 \mathrm{~mm}$ par an caractérisée par une distribution unimodale.

\section{Stations d'échantillonnage}

Les stations ont été choisies en fonction de la proximité des activités anthropiques. Ainsi, les stations ont été sectionnées dans les zones suivantes: une zone qui regroupe les stations proches des champs de coton, une zone qui englobe les stations proches des habitations et une zone qui concerne les stations éloignées de toutes activités anthropiques. Au total, 12 stations ont été choisies dans les ruisseaux du cours moyen de la rivière Alibori (Figure1). Le Global Positioning System (GPS) a été utilisé pour la prise des coordonnées géographiques de chaque station.

\section{Mesure des paramètres physico-chimiques de l'eau}

La mesure in situ des paramètres physico-chimiques de l'eau à savoir la température, la transparence, le $\mathrm{pH}$ et la conductivité a été réalisée au niveau de chaque station d'échantillonnage. Ces mesures ont été effectuées en profondeur de l'eau. La température de l'eau et la conductivité ont été déterminées à l'aide d'un conductimètre multimètre portable Pioneer 30 de Radiometer Analytical. Le $\mathrm{pH}$ a été mesuré avec un $\mathrm{pH}-$ mètre portable Pioneer 10 de Radiometer Analytical. Un disque de Secchi muni d'une corde graduée est utilisé pour la mesure de la transparence de l'eau et de la profondeur des ruisseaux.

Des échantillons d'eau ont été effectués pour l'analyse des nutriments. Des prélèvements d'eau ont été effectués au niveau de chaque station dans des bouteilles en plastique de $500 \mathrm{ml}$ et conservés dans une glacière contenant de glace pour leur acheminement au laboratoire. $\mathrm{La}$ détermination de la concentration en sels dissous des échantillons d'eau prélevés a été réalisée au Laboratoire Hydrobiologie Appliquée de la Faculté des Sciences et Techniques de l'Université d'Abomey-Calavi au moyen d'un spectrophotomètre de marque SHIMADZU UV-1205. Les nutriments mesurés sont les nitrates, les nitrites, 
l'ammonium, l'azote total et le phosphore total.

Un volume de $500 \mathrm{ml} \mathrm{d}$ 'eau a été prélevé dans un contenant de $500 \mathrm{ml}$ en plastique opaque pour le dosage de la chlorophylle a. Il a été utilisé un fluorimètre Sequoia-Turner pour doser la chlorophylle a dans les prélèvements d'eau des différentes stations. Ces dosages ont été réalisés dans le même laboratoire que ceux des sels dissous.

\section{Echantillonnage des macroinvertébrés}

Les macroinvertébrés benthiques ont été échantillonnés au niveau des 12 stations en utilisant les méthodes de Adandédjan (2012). Ils ont été prélevés à l'aide d'un filet troubleau. Cet engin, muni d'un collecteur situé à la base du filet, permet de récolter les organismes après qu'il soit trainé au fond de l'eau à l'aide de sa manche. A chacune des stations, les organismes recueillis dans le filet ont été renversés dans des bocaux. La matière obtenue a été lavée sur l'eau du ruisseau et/ou de la rivière puis filtrée sur un tamis à mailles de $500 \mu \mathrm{m}$. La faune ainsi récoltée, mélangée aux débris végétaux et à la vase, a été mise dans des boîtes en plastique et conservée à l'aide du formol à $5 \%$. Dix coups de filet troubleau ont été donnés à chaque station. Les macrophytes de la berge (deux ou trois plantes) au niveau de chaque station ont été également prélevés à la main pour l'échantillonnage des invertébrés vivant sur leurs racines. La procédure précédente a aussi été adoptée pour leur conservation (Adandédjan, 2012).

Au laboratoire, les organismes ont été triés station par station. Le tri s'est déroulé sous une loupe binoculaire. La détermination taxonomique a été opérée avec les clés : Flore et faune aquatiques de l'Afrique sahélosoudanienne (Durand et Lévêque, 1981); Aquatic entomology (McCafferty, 1981); Invertébrés d'eau douce (Tachet et al., 2000).
La détermination s'est achevée par la conservation des organismes dans l'alcool.

\section{Etudes du peuplement}

L'organisation et la distribution des peuplements ont été mesurées par les calculs de la richesse, l'abondance, l'indice de diversité de Shannon ( $\left.H^{\prime}\right)$ et l'indice d'Equitabilité (E) de Piélou (Adandédjan, 2012).

Pour caractériser les communautés benthiques par les groupes fonctionnels alimentaires présents, les classifications trophiques de Cummins et Klug (1979) et Cummins et Wilzbach (1985) qui ont défini cinq groupes fonctionnels alimentaires (les fragmenteurs, les filtreurs-collecteurs, les rassembleurs-collecteurs, les racleursbrouteurs et les prédateurs) ont été utilisées.

\section{Analyses statistiques}

Les variations spatiales des communautés macrobenthiques des ruisseaux ont été déterminées à l'aide de la carte autoorganisatrice de Kohonen ou « Self Organizing Maps (SOM)» (Kohonen, 2001). L'estimation des erreurs de quantification et de topographie pour différentes tailles de carte de Kohonen (nombre d'unités ou de cellules) nous a permis de choisir une carte de 16 neurones parce qu'elle a présenté la valeur minimale à la fois pour la quantification et la topographie. Une fois la carte de Kohonen obtenue, un algorithme d'Analyse de Classification Hiérarchique (ACH) basée sur la méthode Ward comme critère d'agrégation et la distance Euclidienne a permis ensuite de mettre en évidence des assemblages des objets réels sur la carte (Park et al., 2003). Ces regroupements se sont faits sur la base des similarités entre les stations projetées dans les cellules de la carte SOM. Cet algorithme a été exécuté grâce à la SOM Toolbox (version 2) pour Matlab® (Edia, 2008). 
Les moyennes des paramètres physico-chimiques ont été déterminées et testées avec ANOVA. Le test non paramétrique de Kruskal-Wallis a été employé pour tester la variabilité des paramètres biotiques et abiotiques entre différents groupes de stations définis. Aussi le test de Mann-Whitney a permis de situer les différences entre les groupes. Tous ces différents tests ont été réalisés à l'aide du logiciel STATISTICA version 7.5.

\section{RESULTATS}

\section{Paramètres environnementaux}

Le Tableau 1 présente les paramètres environnementaux et biologiques étudiés. Pour le taux de chlorophylle a, de phosphate total, l'ammonium et nitrates, aucune différence significative $(p>0,05)$ n'a été notée entre eux suivant les stations.

\section{Composition taxonomique du peuplement}

Le Tableau 2 présente la liste des différents taxons de macroinvertébrés benthiques. L'étude a permis de recenser 25 espèces d'invertébrés aquatiques appartenant à 16 familles et 7 ordres. Cinq (5) taxons dont la plupart sont essentiellement des insectes, ont été indéterminés au niveau du genre et/ou de l'espèce et 1 taxon au niveau de la famille. Trois classes de macroinvertébrés benthiques ont été récoltées au cours de l'étude. Il s'agit des Insectes (5 ordres, 12 familles et 20 espèces), des Gastéropodes (1 ordre, 3 familles et 4 espèces) et des Lamellibranches (Bivalves) ( 1 ordre, 1 famille et 1 espèce).

L'entomofaune a été très riche avec les Coléoptères qui ont renfermé 4 familles et 9 espèces, soit $23 \%$ des familles et $36 \%$ de la richesse totale. Ils ont été suivis des Diptères et des Hémiptères avec chacun 3 familles et 3 espèces constituant ainsi $18 \%$ des familles et $12 \%$ de la richesse spécifique. Les Odonates (2 familles et 4 espèces) et les Ephéméroptères (1 famille et 1 espèce) ont été aussi des ordres importants de cette entomofaune.

La malacofaune a renfermé au total 4 familles dont 3 familles de Gastéropodes et une des Bivalves constituant respectivement 18 et $6 \%$ des familles puis 16 et $4 \%$ de la richesse spécifique du bassin de la rivière durant l'étude.

$\mathrm{Du}$ point de vue abondance, quatre ordres ont été prépondérants. Les gastéropodes ont été les ordres les plus importants constituant ainsi 48,2\% de l'abondance totale. Suivent les Diptères et les Coléoptères qui ont été constitués respectivement de $19,8 \%$ et $16,66 \%$ de l'effectif total des organismes. Les Odonates avec $11,2 \%$ du nombre total des individus ont été un autre ordre important de la macrofaune récoltée.

Quant aux familles, ce sont les Thiaridae constituées de $40,2 \%$ de l'effectif total qui ont été les plus abondantes. Viennent ensuite les Chironomidae et les Gomphidae avec respectivement $19,5 \%$ et $10,9 \%$ du nombre total des individus récoltés. Les Lymnaeidae, Hydrophilidae, Dytiscidae et Chrysomelidae ont été aussi des familles importantes de la macrofaune des ruisseaux de la rivière. Neuf familles de cette faune ont moins de $5 \%$ d'abondance relative.

\section{Classification hiérarchique}

La Figure 2 donne le résultat de l'Analyse de Classification Hiérarchique qui a permis de regrouper les 16 cellules de la carte de Kohonen en quatre groupes (I à IV).

Chaque groupe, représenté sur la carte à la Figure 3 avec le même motif, a été constitué d'échantillons ayant des compositions taxonomiques semblables. Cette analyse a révélé une répartition spatiale indiquant que les différentes stations ont eu en commun une grande partie de la faune benthique mais se sont différenciées par un nombre limité de taxons qui ont été 
caractéristiques de chaque site. Les groupes II et IV, riches en taxons se sont isolés des groupes I et III plus pauvres en taxons. Le groupe I est composé de 3 échantillons. Le second groupe a renfermé 2 échantillons. Quant au troisième, il a été composé de 5 échantillons. Le quatrième groupe a rassemblé 2 échantillons. La classification sur la base des facteurs environnementaux révèle que le groupe I est caractérisé par des valeurs élevées de $\mathrm{pH}$ et de la concentration en azote total. Le groupe II a été défini essentiellement par la concentration en sels phosphatés. Quant aux échantillons du groupe III, ils se sont distingués essentiellement par les températures importantes. Enfin le groupe IV a regroupé des échantillons issus des stations plus profondes avec des valeurs élevées de la transparence de l'eau.

\section{Structure du peuplement}

$\mathrm{Du}$ point de vue fonctionnel, les collecteurs-rassembleurs et les prédateurs ont été les groupes les plus importants dans le milieu d'étude constitués respectivement de $40 \%$ et $35 \%$ de l'effectif total. Les taxons racleurs occupent la troisième place avec $20 \%$ de l'effectif des groupes fonctionnels alimentaires. Les filtreurs-collecteurs ont été les groupes les moins représentés dans le bassin pendant la période d'étude avec un effectif de $5 \%$.

La Figure 4 a illustré la richesse taxonomique des différents groupes définis par la SOM. Le nombre de taxons a varié de 2 taxons pour le groupe I qui a totalisé la plus faible richesse ( 2 espèces) à 8 taxons pour le groupe II. Le groupe III a 5 espèces et le groupe IV en a 8 . Le nombre de taxons diffère d'un groupe à l'autre (Kruskal-Wallis, p < $0,05)$. Le nombre de taxons du groupe I a été différent de celui du groupe III (Mann-
Whitney, $\mathrm{p}<0,01)$. Le nombre de taxons du groupe IV a été différent de celui des groupes II et III (Mann-Whitney, $\mathrm{p}<0,05$ ), tandis qu'il n'y a pas eu de différence significative entre les groupes I et IV puis entre les groupes I et II (Mann-Whitney, $\mathrm{p}>0,05$ ).

Le profil de distribution des 25 taxons de macroinvertébrés effectué au moyen de SOM (Figure 5) montre que le groupe I a été caractérisé significativement par des taxons dont les noms sont les suivants : Chironomus sp. et Paragomphus sp.. S'agissant du groupe II, il a été distingué par la présence d'espèces que sont Cricotopus sp., un taxon de Ceratopogonidae, un taxon de Nepidae, Mesovelia sp., Paragomphus hajeni, Lymaea sp., Melanoides sp. et une espèce indéterminée de Coléoptères. Quant au groupe III, il a été démarqué par des taxons comme Donacia sp., Amphiops senegalensis, Ictinogomphus sp., Pseudocleon sp., Berosus striatus, et une seule espèce indéterminée de Coléoptères Hydrophilidae. Le dernier groupe (IV) a été constitué des taxons à savoir Microvelia sp., Dytiscus sp., Hydaticus sp., Rhantaticus congestus, Pseudocloeon sp., Melanoides tuberculata, Gyraulus sp. et la seule espèce de Bivalves.

L'indice de diversité de Shannon H' a varié entre 0,00 bits aux stations Gban, Nawa puis Sori et 2,48 bits à la station Zanr. Sur l'ensemble, cet indice n'a montré aucune variabilité (test de Kruskal-Wallis: $p>0,05$ ) entre les stations. De même, l'Equitabilité a présenté une gamme de valeurs qui ont oscillé entre 0,00 aux stations Gban, Nawa puis Sori et 0,94 à la station Kpto. Aucune variation spatiale significative n'a été notée selon le test de Kruskal-Wallis ( $\mathrm{p}>0,05)$. 


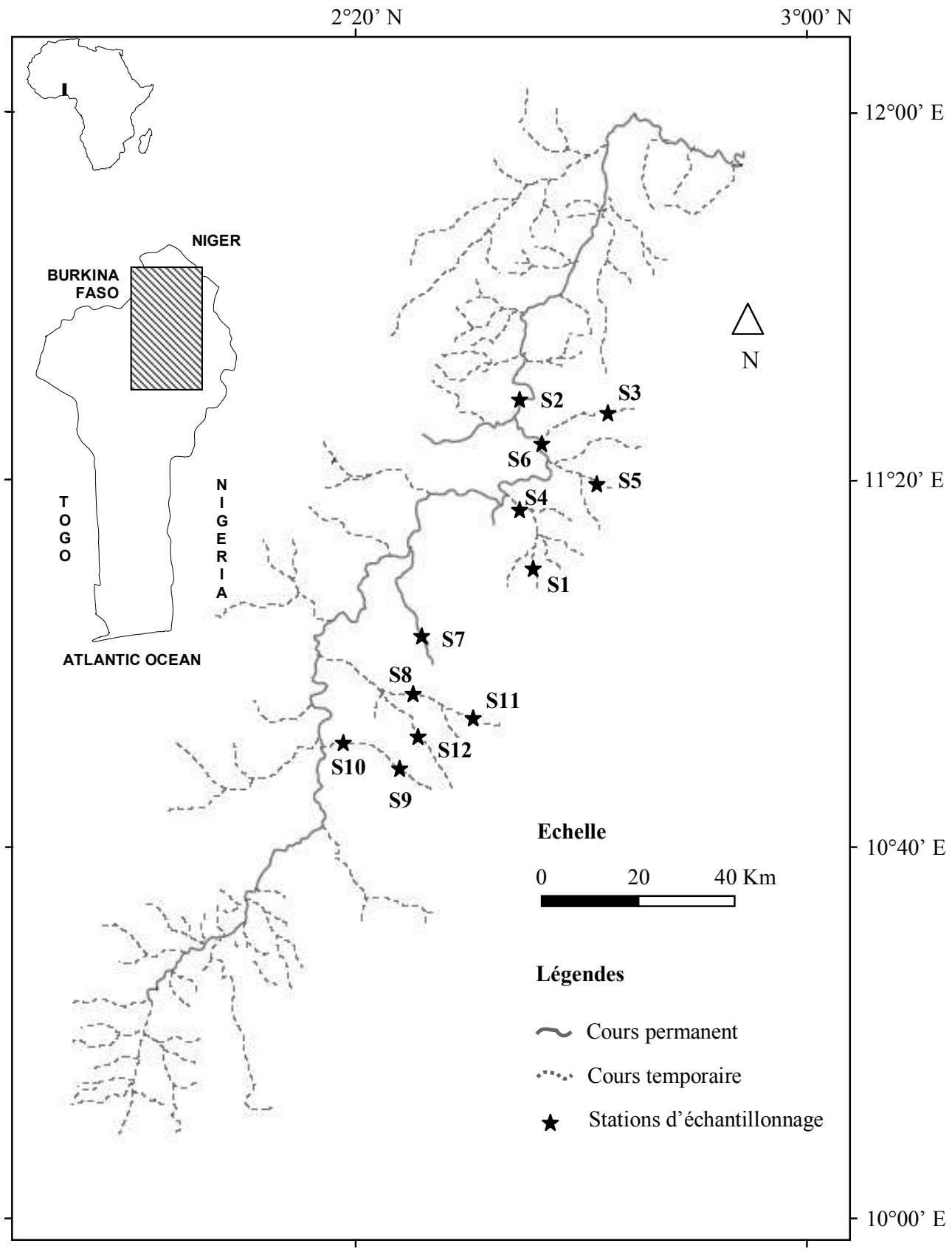

Figure 1: Bassin de la rivière Alibori et stations d'échantillonnage.

$S 1=$ Tissarou (Tiss) $S 2=$ AliboriK (Albo), S3=Bagorro (Bago); S4=Gambanè (Gamb), S5=Zanroua (Zanr), S6= Kpedekonongou (kpto=), S7= Gbandarou (Gban), S8=Nawari (Nawa), S9=Kantakparal (Kant), S10= Sori (Sori), S11= Ouèrè (Ouèrè), S12= Kantakpara 2 (Kam). 
(A)

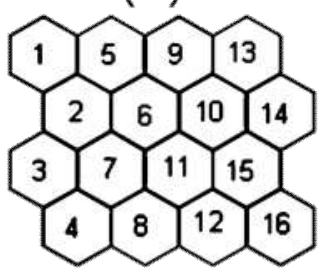

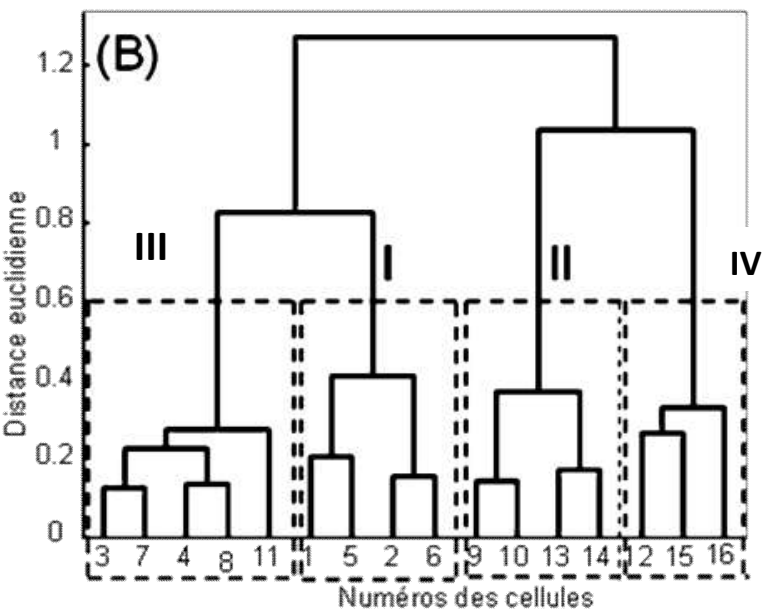

Figure 2: Carte de Kohonen (A) et classification hiérarchique des cellules de la carte de Kohonen (B) dans les ruisseaux de la Rivière Alibori sur la base de la richesse taxinomique avec la méthode Ward et la distance Euclidienne comme distance d'assemblage. Les nombres (1 à 16) correspondent aux numéros de cellules de la carte de Kohonen; les chiffres romains (I à IV) représentent les groupes retenus.

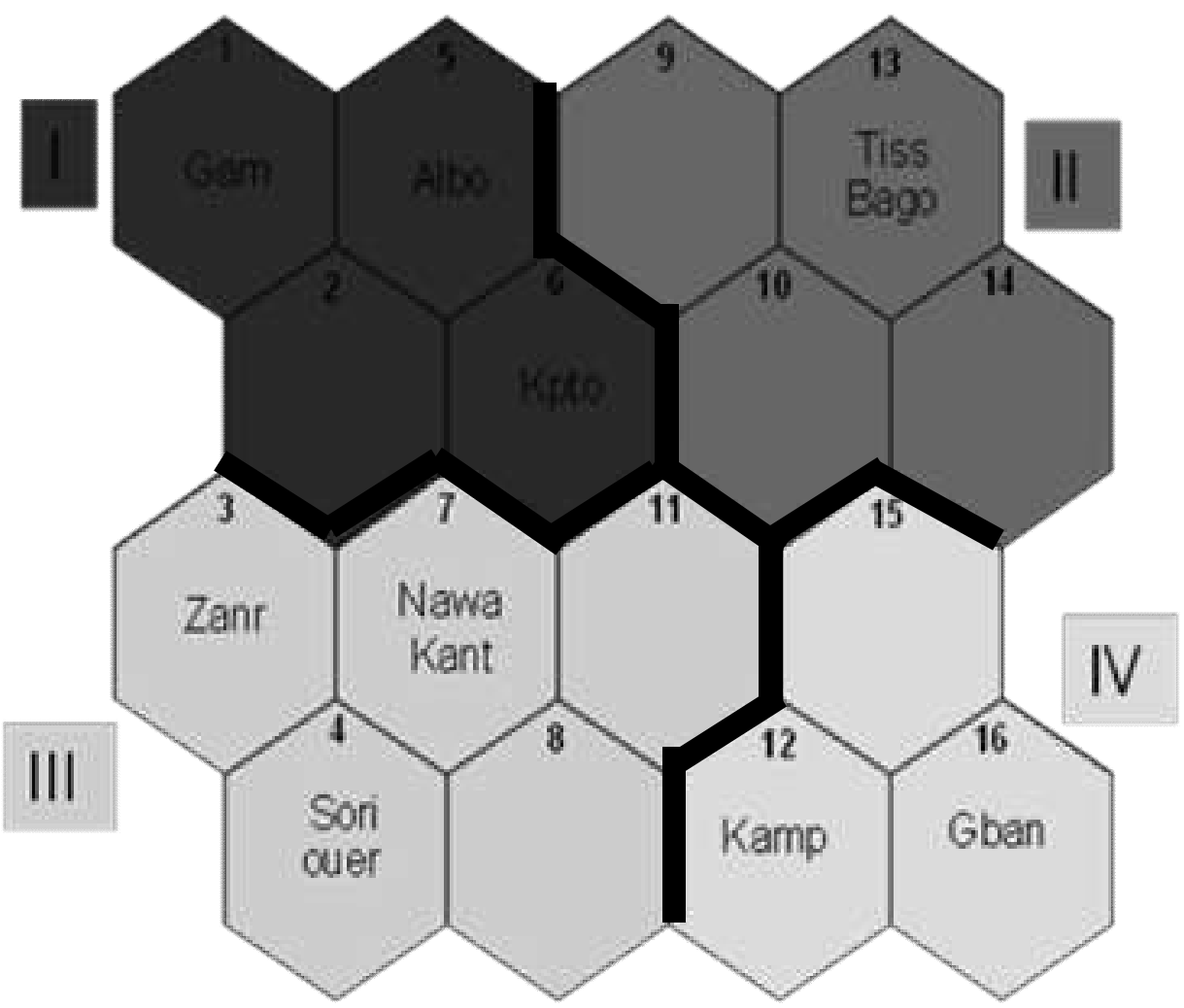

Figure 3 : Distribution des échantillons des ruisseaux du bassin de la Rivière Alibori sur la carte de Kohonen à partir de la composition taxinomique. Les groupes définis sont numérotés de I à IV; Tiss = Tissaroou, Albo $=$ Alibori K, Bago $=$ Bagorro, Gam $=$ Gambanè, Kpto $=$ Kpédékongou, Gban $=$ Gbandarou, Zanr $=$ Zanroua, Kant $=$ Kantakparal, Kamp =Kantakpara 2, Naw =Nawari, ouer $=$ ouerè, les nombres de 1 à 16 au sommet des cellules représentent leurs numéros respectifs. 


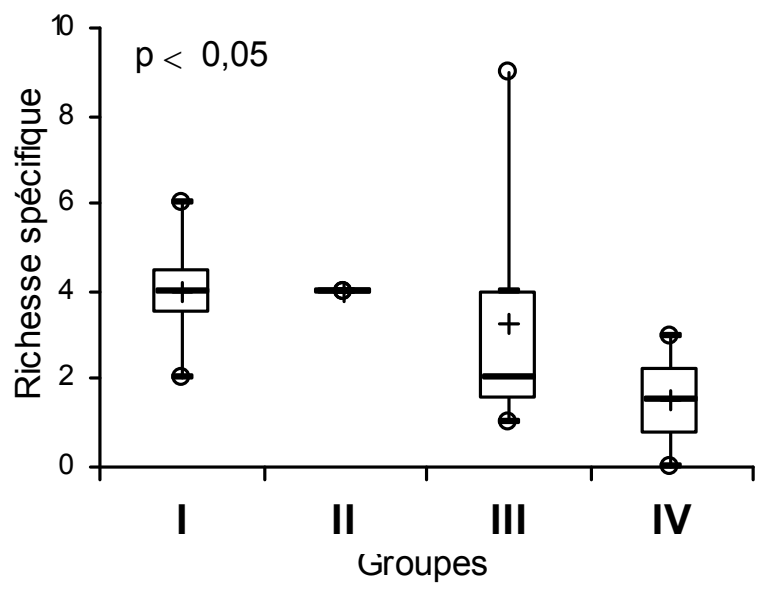

Figure 4 : Richesse taxonomique entre les différents groupes des ruisseaux du bassin de la rivière Alibori définis par la SOM.

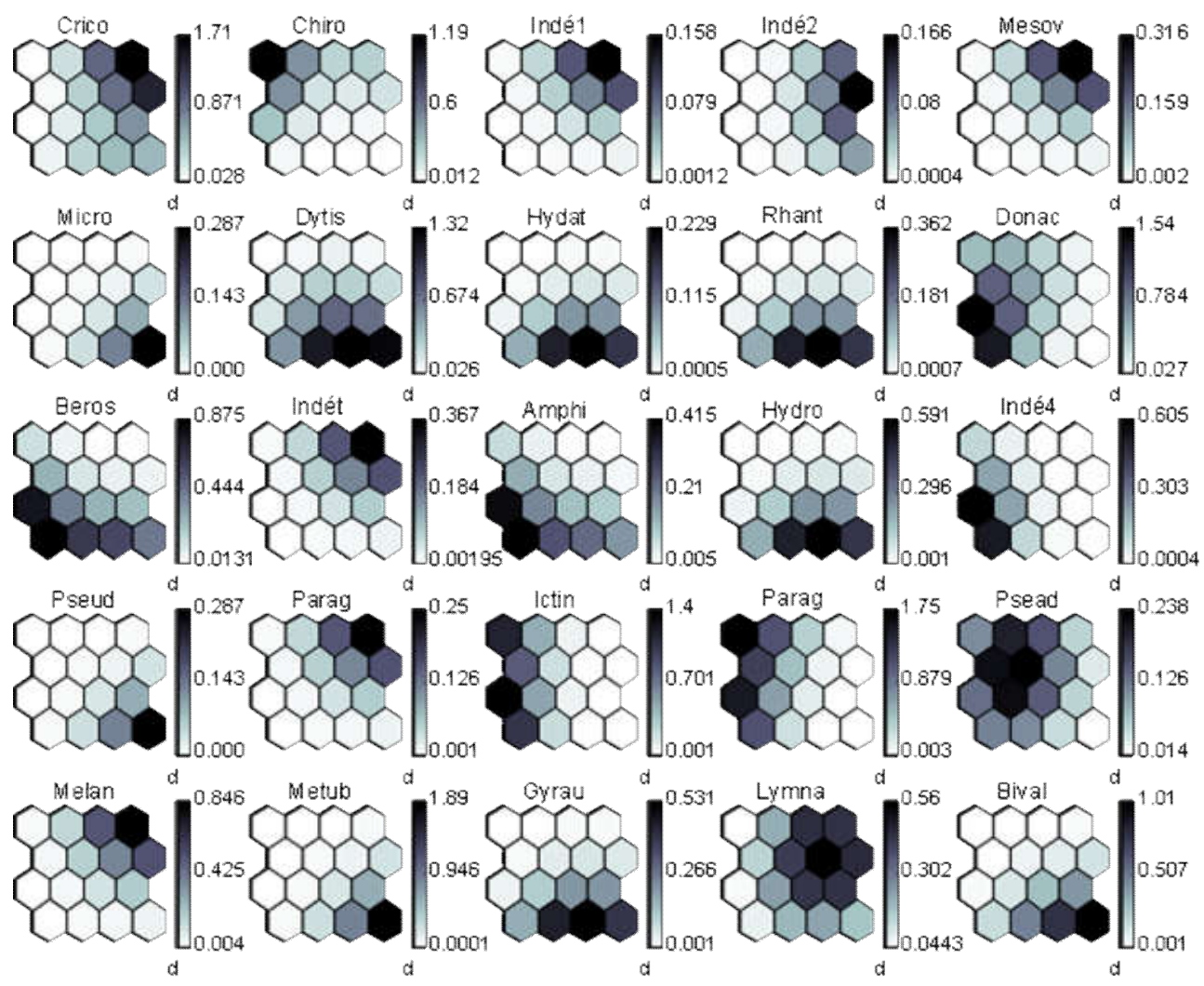

Figure 5 : Profil de distribution de chaque taxon des macroinvertébrés des ruisseaux du bassin de la Rivière Alibori sur la carte de Kohonen basée sur les données de présence-absence. Couleur foncée $=$ fréquence élevée; couleur claire = faible fréquence voir absence; $d=$ échelle. 
Tableau 1 : Synthèse des tests de comparaison (ANOVA) sur les variations physico-chimiques.

\begin{tabular}{|c|c|c|c|}
\hline Paramètres & Moyennes & $\mathbf{p}$ & Significativité \\
\hline Transparence (cm) & 16 & 0,0002 & $* * *$ \\
\hline Profondeur(m) & 0,17 & 0,0002 & $* * *$ \\
\hline $\mathrm{T}^{\circ} \mathrm{C}$ & 28 & 0,00001 & $* * *$ \\
\hline $\mathrm{pH}$ & 8 & 0,0012 & $* * *$ \\
\hline Conductivité $(\mu \mathrm{S} / \mathrm{cm})$ & 138 & 0,00001 & $* *$ \\
\hline Chlorophylle $a$ & 1192,81 & 0,196 & NS \\
\hline $\mathrm{PO}_{4}(\mathrm{mg} / \mathrm{L})$ & 1,6 & 0,363 & NS \\
\hline NKT (mg/L) & 1,48 & 0,008 & $* *$ \\
\hline $\mathrm{NH}_{4}(\mathrm{mg} / \mathrm{L})$ & 0,23 & 0,36 & NS \\
\hline $\mathrm{NO}_{3}(\mathrm{mg} / \mathrm{L})$ & 1,37 & 0,048 & $*$ \\
\hline $\mathrm{NO}_{2}(\mathrm{mg} / \mathrm{L})$ & 0,04 & 0,201 & NS \\
\hline
\end{tabular}


Tableau 2: Liste des taxons de macroinvertébrés benthiques échantillonnés du bassin versant de la rivière Alibori.

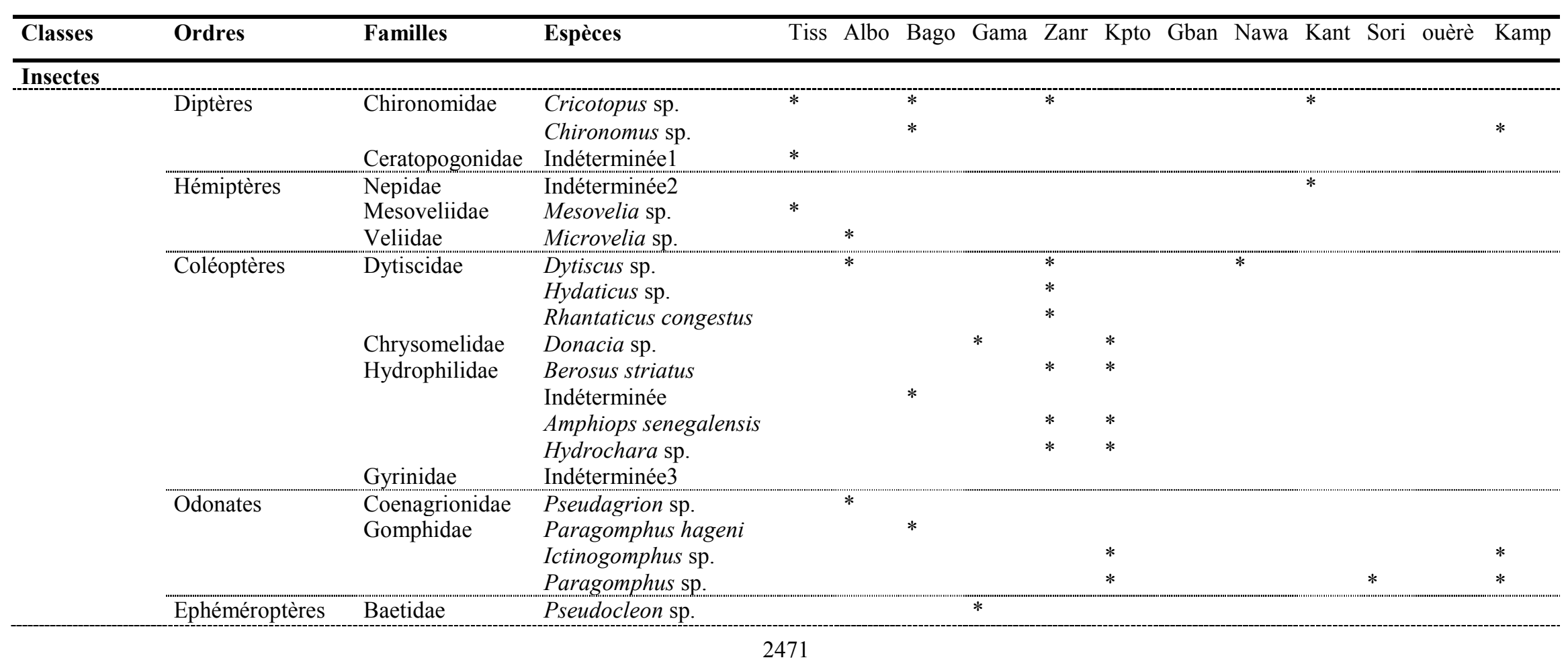


T. M. AGBLONON HOUELOME et al. / Int. J. Biol. Chem. Sci. 10(6): 2461-2476, 2016

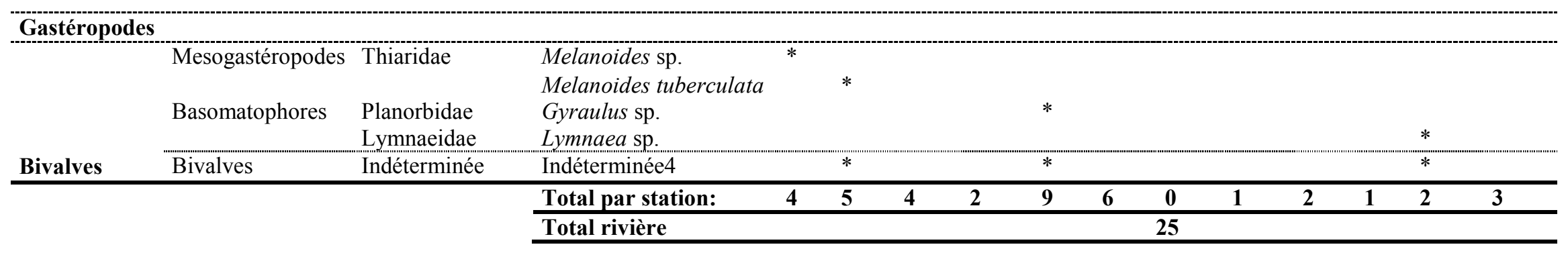




\section{DISCUSSION}

Les valeurs de $\mathrm{pH}$ et de conductivité observées pourraient faire penser à une eau de bonne qualité (Nounon et al., 2015). Néanmoins la faible transparence de l'eau observée ne pourra pas soutenir cette thèse d'autant plus qu'un tel phénomène explique souvent une eau trop chargée de matières. Cette turbidité observée pourrait être liée à la position des champs par rapport aux ruisseaux, qui par le phénomène de lessivage chargent les eaux de rejets agricoles qui sédimentent dans les ruisseaux une fois l'eau retirée. Les engrais sont ainsi véhiculés par ruissellement et drainage vers la rivière (Aboudou, 2010). En effet, la plupart des nutriments à savoir le phosphore total, l'ammonium et les nitrates dépasse les normes au niveau de certaines stations (Dovonou et al., 2011). Les valeurs élevées de l'ammonium au niveau de certaines stations, premier indicateur de la pollution de l'eau, et les taux alarmants du phosphore à certains endroits sont des situations qui expliquent ce lessivage des terres agricoles dans la zone d'étude. De plus, l'obtention d'une forte biomasse algale corrélée positivement au taux de phosphore traduit une eutrophisation des eaux. C'est un résultat qui est similaire à ceux déjà obtenus au cours de certaines études dans les plans d'eau du Nord-Bénin comme le cas de la rivière Pendjari par Ahouansou-Montcho (2011), qui rapporte que l'importance de la biomasse algale dans une rivière est due au taux du phosphore total. Les mêmes remarques ont été faites même en lagune et spécifiquement dans les lagunes Côtière et de Porto-Novo où Adandédjan (2012) avait trouvé une forte corrélation positive entre les concentrations des sels phosphatés et la couverture végétale des plans d'eau. Les faibles transparences obtenues s'expliqueraient alors par une eutrophisation des eaux qui se traduit par une croissance excessive des algues et un appauvrissement de l'eau en oxygène, paramètre nécessaire à la vie des organismes. La conséquence est l'élévation du taux de mortalité chez certains organismes aquatiques. Dovonou et al. (2011) identifiaient aussi l'accumulation des engrais notamment le phosphore dans les sols agricoles comme la cause probable de contamination des rivières en milieu rural et le phosphore en excès provoque l'eutrophisation. La diversité de la macrofaune benthique récoltée est marquée par l'importance des Gastéropodes dominés par la famille des Thiaridae tant en richesse qu'en effectif. Cette observation met en exergue l'importante quantité de matières organiques et l'existence de plantes aquatiques dont se nourrissent ces familles à certaines stations. La faune des ruisseaux est principalement composée de Mollusques et ceux-ci présentent une faible richesse taxonomique. Ce résultat a été déjà trouvé par Aboudou (2010) au cours de la caractérisation de la diversité et de la structure des organismes benthiques dans le bassin cotonnier du Nord-Bénin. Ceci pourrait être dû à l'important couvert végétal sur toute la longueur des ruisseaux car selon Adandédjan et al. (2012), ce facteur contribuait à augmenter l'hétérogénéité des habitats qui influent sur la composition des communautés benthiques. La diversité des Diptères observée et surtout des familles de Chironomidae, taxons polluo-résistants (Gnohossou, 2006; Adandédjan, 2012) aux stations situées surtout en aval et à proximité des champs révèle une accumulation de nutriments dans l'écosystème. En effet, la présence marquée de ces familles plaide en faveur de la présence d'un milieu enrichi en matières organiques, conséquences d'intenses activités anthropiques (Carvalho et al., 2006). Ces activités installées à proximité des écosystèmes perturbent les communautés benthiques et contribuent à la diminution de la richesse spécifique et même la répartition des espèces (Silveira et al., 2006). Ces résultats sont encore attestés par la faible présence d'Ephéméroptères et l'absence d'Hétéroptères et de Trichoptères. En effet, ces taxons surtout polluo-sensibles dont la présence dans un milieu est indicatrice de la bonne qualité des eaux ne pourraient proliférer dans un milieu 
perturbé. Ces organismes sont en effet réputés vivre dans des milieux bien oxygénés et de bonne qualité (Carvalho et al., 2006). Des travaux similaires indiquent par ailleurs, que la richesse spécifique des Ephéméroptères baisse avec l'eutrophisation des bassins (Sanogo et al., 2014). D'autre part, la prolifération des collecteurs-rassembleurs et le faible nombre de filtreurs expliquent une abondance de matières organiques accumulées dans la rivière et ses affluents. Il est démontré que lorsque la charge organique devient importante des espèces polluo-sensibles disparaissent pour laisser place aux espèces plus résistantes comme les Diptères Chironomidae et certains Coléoptères comme les Chrysomelidae (Edia, 2008; Adandédjan et al., 2012). Cette observation qui justifie les faibles valeurs de l'indice de Shannon sur l'ensemble du bassin. La situation s'accorde parfaitement aux faibles transparences enregistrées dans les ruisseaux d'Alibori durant l'étude qui expriment une eau chargée de particules et explique aisément l'importance des racleurs que sont les Gastéropodes à toutes les stations du bassin (Younes-Baraillé et al., 2005). La prépondérance des prédateurs observée aux stations surtout en aval serait liée aux compétitions intra-spécifiques comme des espèces devenaient rares à certaines stations comme Gbandarou, Nawari, Ouèrè et à proximité des champs de coton (Uwadiae, 2010). Cependant, la faible proportion des filtreurs-collecteurs témoigne de la présence d'une eau possédant encore une capacité d'épuration. La structure des peuplements observée révèle deux grandes communautés. Les communautés des groupes II et IV éloignées de zones des champs et des habitations avec des richesses taxonomiques élevées sont formées de stations moins polluées présentant également une bonne valeur de l'indice de l'Equitabilité relativement élevée, ces groupes traduisent un relatif équilibre du milieu à la décrue de la rivière. A l'opposé, les groupes I et III essentiellement constituées de stations plus perturbées à faible diversité.

\section{Conclusion}

La présente étude a permis de recenser dans le bassin versant de la rivière Alibori vingt-cinq (25) taxons d'invertébrés repartis en deux Classes à savoir les Insectes et les Mollusques. La classe la plus diversifiée a été celle des Insectes. La richesse spatiale du bassin a montré une distribution indiquant que les différentes stations ont eu en commun une grande partie de la faune benthique mais se sont différenciées par un nombre limité de taxons qui ont été caractéristiques de chaque site. La température, le $\mathrm{pH}$, la transparence, la conductivité, l'azote total et le phosphore total sont les paramètres environnementaux qui ont discriminé la répartition des organismes dans le bassin. L'examen de la structure a révélé une macrofaune benthique dominée par les collecteurs-rassembleurs et les prédateurs. Deux catégories de communautés ont caractérisé cette structure, la communauté des sites éloignés de zones des champs et des habitations avec des richesses taxonomiques élevées puis celle des zones de production agricole qui ont présenté des diversités faibles. La présence des indicateurs de pollution comme les Chironomidae et les Chrysomelidae et l'absence de taxons surtout polluo-sensibles dans le bassin est l'expression de l'impact des activités agricoles réalisées à côté des stations.

\section{CONTRIBUTIONS DES AUTEURS}

TMAH s'est occupé de la collecte des données physico-chimiques, de l'échantillonnage et de l'identification des macroinvertébrés. DA a procédé à la validation taxonomique des spécimens de macroinvertébrés et a supervisé les analyses statistiques réalisées à l'aide de la carte autoorganisatrice de Kohonen (Self Organizing Maps (SOM). AC a défini et supervisé le protocole de collecte et d'analyse des variables physico-chimiques. IIT a défini et supervisé le protocole d'échantillonnage des 
macroinvertébrés dans les ruisseaux. Il est responsable des aspects liés à la qualité physique et chimique des eaux de la rivière.

$\mathrm{CB}$ a contribué à l'identification de certains groupes de macroinvertébrés, notamment les insectes. IY a défini le protocole général d'analyses statistiques des données, notamment les analyses multivariées. PL a offert le cadre et les équipements nécessaires à la réalisation de cette étude.

\section{REFERENCES}

Aboudou EF. 2010. Caractérisation de la diversité et de la structure des macroinvertébrés benthiques $\mathrm{du}$ bassin cotonnier Béninois: Banikoara, Kandi, Gogounou. Thèse d'Ingénieur Agronome, Université de Parakou, Parakou, p. 94.

Adandédjan D. 2012. Diversité et déterminisme des peuplements de macroinvertébrés benthiques de deux lagunes du Sud- Bénin : la Lagune de Porto-Novo et la Lagune Côtière. Thèse de doctorat, Université d'Abomey-CalaviBénin, Abomey-Calavi, p. 261.

Adandédjan D, Lalèyè P, Gourène G. 2012. Macroinvertebrates communities of a coastal lagoon in southern Benin, West Africa. Int. J. Biol. Chem. Sci., 6(3): 12331252 .

Agbohessi TP, Imorou Toko I, Yabi AJ, Dassoundo CFJ, Kestemont P. 2011. Caractérisation des pesticides chimiques utilisés en production cotonnière et impacts sur les indicateurs économiques dans la commune de Banikoara au nord du Bénin. Int. J. Biol. Chem. Sci., 5(5): 18281841.

Ahounsou Montcho S. 2011. Diversité et exploitation des poissons de la rivière Pendjari (Bénin, Afrique de l'Ouest). Thèse de Doctorat, Université d'AbomeyCalavi, Abomey-Calavi, p. 214.

Bio Bigou LB. 1987. La vallée béninonigérienne du fleuve Niger : Population et développement économique. Thèse de Doctorat (nouveau régime), Université de Bourgogne, Dijon, p. 917.
Carvalho S, Barata M, Pereira F, Gaspar MB, da Fonseca LC, Pousao-Ferreira P. 2006. Distribution patterns of macrobenthic species in relation to organic enrichment within aquaculture earthern ponds. Marine Polluion Bulletin, 52: 1573-1584.

Clarke RT, Furse MT, Gunn RJM, Winder JM, Wright JF. 2002. Sampling variation in macroinvertebrate data and implications for river quality indices. Freshwater Biology, 47: 1735-1751.

Cummins KW, Klug MJ. 1979. Feeding ecology of stream invertebrates. Annual Review of Ecology and Systematic, 10: 147-172.

Dovonou F, Aina M, Boukari M, Alassane A. 2011. Pollution physico-chimique et bactériologique d'un écosystème aquatique et ses risques écotoxicologiques : cas du lac Nokoue au Sud Benin. Int. J. Biol. Chem. Sci., 5(4): 1590-1602.

Edia OE. 2008. Diversité taxonomique et structure des peuplements de l'entomofaune des rivières côtières Soumié, Eholié, Ehania, Noé (Sudest, Côte d'Ivoire). Thèse de doctorat Option : Ecologie et aménagement des écosystèmes aquatiques. Laboratoire d'Ecologie et de Biologie Appliquée, Université d'AboboAdjamè, Abobo-Adjame, p. 153.

Fishar MR, Williams WP. 2008. The development of a Biotic Pollution Index for the River Nile in Egypt. Hydrobiologia, 598: 17-34.

Gnohossou PM. 2006. La faune benthique d'une lagune ouest africaine (le Lac Nokoué au Bénin), diversité, abondance, variations temporelles et spatiales, place dans la chaîne trophique. Thèse de Doctorat, Institut National Polytechnique de Toulouse, Toulouse, p. 169.

Hepp LU, Milesi SV, Restello RM. 2010. Effects of agricultural and urban impacts on macroinvertebrates assemblages in streams (Rio Grande do Sul, Brazil). Socieda de Brasileira de Zoologia , 1(27): 106-113 
Kohonen T. 2001. Self-organizing Maps (3rd edn). Springer: Berlin.

Le Barbé L, Ale G, Millet B, Texier H, Bore L. 1993. Monographie des ressources en eaux superficielles de la République du Bénin. ORSTOM, Paris, p.540.

Leigh C, Burford MA, Roberts DT, Udy JW. 2010. Predicting the vulnerability of reservoirs to poor water quality and cyanobacterial blooms. Water Research, 44: 4487-4496.

Silveira MP, Buss DF, Nessimian JI, Baptista DF. 2006. Spatial and temporal distribution of benthic macroinvertebrates in a southeastern brazilian river. Braz. J. Biol., 66(2B): 623-632.

Noumon CJ, Mama D, Dedjiho CA, Agbossou E, Ibouraima S. 2015. Évaluation de la qualité physico-chimique et du risque d'eutrophisation de la retenue d'eau de Kogbétohouè (Sud-Bénin). Journal of Applied Biosciences, 85: 7848-7861.

Park YS, Céréghino R, Compin A, Lek S. 2003. Applications of artificial neural networks for patterning and predicting aquatic insects species richness in running waters. Ecological Modelling, 160: 265280.

Sanogo S, Kabré JAN, Cecci P, 2014. Inventaire et distribution spatio-temporelle des macroinvertébrés bioindicateurs de trois plans d'eau du bassin de la Volta au Burkina Faso. Int. J. Biol. Chem. Sci., 8(3): 1005-1029.

Sass LL, Bozek MA, Hauxwell JA, Wagner K, Knight S. 2010. Response of aquatic macrophytes to human land use perturbations in the watersheds of Wisconsin lakes, U.S.A. Aquatic Botany, 93: 1-8.

Touzin D. 2008. Utilisation des macroinvertébrés benthiques pour évaluer la dégradation de la qualité de l'eau des rivières au Québec. Thèse d'Ingénieur Agronome, Faculté des sciences de l'agriculture et de l'alimentation, Université Laval, Laval, 40 p.

Uwadiae RF. 2010. Macroinvertebrates functional feeding groups as indices of biological assessment in a tropical aquatic ecosystem: implications for ecosystem functions. New York Science Journal, 3(8).

Younes-Baraillé Y, Garcia XF, Gagneur J. 2005. Impact of the longitudinal and seasonal changes of the water quality on the benthic macroinvertebrate assemblages of the Andorran strezams. C.R. Biologies, 328: 963-976. 